\title{
The role of the standard in an auditory amplitude discrimination experiment*
}

\author{
GLENIS R. LONG \\ York College of the City University of New York, Jamaica, New York 11432
}

\begin{abstract}
The role of the standard in a fixed-standard experiment of auditory amplitude discrimination was examined by varying the proportion of trials on which it was presented. The results suggest that the Os may use different perceptual strategies, depending on the availability of the standard. While they did better on trials containing a standard regardless of the proportion of such trials, their accuracy on both "standard-present" and "standard-absent" trials was positively related to the probability of the particular type of trial. These results conflict with the claim of advocates of the roving-standard paradigm that the $O$ in the fixed-standard experiment does not use the standard but, rather, compares the variable with a long-term referent built up over many trials. The introduction of a second pair of tones, making it a random-standard experiment, produced considerable response bias tied to the stimulus level and to an overall deterioration in performance.
\end{abstract}

A basic task in a variety of psychophysical experiments is the "delayed comparison" paradigm. A standard stimulus is followed, after some delay, by a comparison stimulus. The $\mathrm{O}$ must then make some judgment about the relation between these two stimuli, e.g., "same" or "different," "stronger" or "weaker," "same" or "softer," etc. Such an experiment often consists of several sessions, each of which contains a series of delayed comparison trials in which the standard stimulus is a constant value on all trials while the comparison stimulus varies randomly between two possible values: it is either the same as the standard or some constant deviation from it on each trial.

Some parameters of this type of perceptual task which have been shown to be important are: the durations of the standard and the delay between the standard and comparison stimuli. The discovery that the O's discrimination. "sensitivity," generally increases as the duration of the standard increases (Wickelgren, 1966, 1969) and as the interstimulus interval is diminished (Harris, 1952: Kinchla \& Smyzer, 1967; Wickelgren, 1966, 1969) has led to theoretical proposals regarding an "input" or "acquisition process." whereby the $O$ develops an adequate internal representation of a stimulus over some period of time, and a "memory" process," by which he maintains a "memory" of this representation during the interstimulus delay, in order to make the delayed comparison. Other research by this author (Long. 1971) suggests that the "acquisition process" may continue for a set interval after stimulus onset. even if the physical stimulus terminates prior to

*This paper is based upon part of a dissertation submitted to Princeton University in partial fulfillment of the requirements for the PhD degree. The author wishes to thank Dr. R. A. Kinchla. Dr. E. G. Wever. and Dr. J. Darley for their guidance. Dr. N. Durlach is to be thanked also for several useful comments on the paper. The research was supported by a PHS Grant 5 SO5 l:R 7057-04. that interval. In such cases, the "memory" process may not start with the offset of the standard but, rather, at the conclusion of the acquisition phase. This paper describes some experiments which attempt to determine the extent to which the comparison on any one trial is based on the particular standard presented on that trial.

When an $\mathrm{O}$ makes a discrimination judgment in a delayed comparison experiment, he must compare a sensory representation of the "comparison" stimulus with some stored (remembered) representation. This memory representation may be of at least two possible types: a temporary, and rapidly decaying, representation of the standard stimulus presented on a particular trial, or a relatively stable representation which has been built up from the effect of many previous trials and is e ssentially independent of the standard stimulus presented on any one trial.

In an attempt to relate findings on intensity resolution from a variety of experimental paradigms (detection, discrimination, identification, magnitude estimation, and category scaling), Durlach and Braida (1969) developed a theory in which it is assumed that the $O$ has two such memory operating modes: a "sensory-trace mode" and a "context-coding mode." The 0 , using the "sensory-trace" mode, is assumed to try to maintain a "trace" or "image" of the standard stimulus. This trace is thought to decay over time and with interference. In the context-coding mode, the $O$ is thought to evaluate the loudness of a sound by comparing it with the total context of sounds in the experiment. It is assumed that this representation is formed imperfectly immediately after the sensation and remembered perfectly so that the amount of error ("noise") in the representation is a function of the range of the stimuli in the experiment but independent of time and interference.

It has most generally been assumed that the trace mode is used whenever a standard or comparison 
stimulus is available (two-interval paradigms), whereas the context mode is used in identification (absolute judgment) experiments where only one stimulus is presented on each trial (one-interval paradigm). Harris (1952), Wickelgren (1966, 1969), and others have questioned this view. They claim that the context mode will be used in two-interval paradigms in which the standard is always presented first unless the overall level of the pair of tones constituting a single trial is varied randomly from trial to trial ("roving-standard" discrimination experiments). This claim is based on Harris's finding that pitch discrimination deteriorated little with increases in the interstimulus interval in a "iixed-standard" (constant level) experiment but deteriorated markedly in a roving-standard experiment when the interstimulus intervals were greater than $1 \mathrm{sec}$. They claim that this indicates that the comparison stimuli in the fixed-standard experiment were not compared with a decaying trace of a particular standard but with a nondecaying stable representation. Such claims have led to the use of a roving-standard paradigm in most experiments in which it is important that the $O$ compare the comparison stimulus with some representation of the standard. Harris's conclusions that a nondecaying trace was used in the fixed-standard experiment can, however, be questioned since he used a relatively coarse measurement (the "difference limen," DL) based on a small amount of data.

Durlach and Braida (1969) also suggest that the context mode is used in fixed-standard amplitude discrimination experiments. This is because they believe that only when a roving standard is used is the noise due to context greater than that due to time. This claim is not, however, essential to their theory, because it is based on an assumption that both context noise and trace noise go to a minimum of zero. An alternative assumption, that one or both reach some minimal value above zero, seems just as plausible. If minimal context noise is greater than minimal trace noise, the trace mode would be used in fixed-standard experiments until the interstimulus interval was so long that the trace noise was greater than minimal context noise.

Support for the alternative assumption comes from research by Kinchla and Smyzer (1967). They found decrements in amplitude discrimination performance in a fixed-standard amplitude discrimination experiment with increases in the interstimulus interval to $2 \mathrm{sec}$. The measure used, $\mathrm{d}^{\prime}$, a signal detection theory measure which separates sensitivity from response bias, is much more sensitive than DL. Durlach and Braida applied their theory to Kinchla and Smyzer's data and found that it could be argued that the change from trace to context mode occurred at about $3.5 \mathrm{sec}$. They admit that this is contrary to their theory and hypothesize that this is due to a decay in the context storage over time. It could, however, be interpreted as evidence that comparison judgments in an amplitude discrimination task are based on a trace of the standard. It is still an open question as to which mode is used for pitch discrimination.

Additional evidence that minimal context noise may be larger than that predicted by Durlach and Braida comes from a study by Viemeister (1970). He compared performance on a two-interval forced-choice and an identification task and found that the context-coding variance was larger than would be predicted from the small range of intensities used.

Another way to determine the nature of the comparison process in a discrimination experiment would be to examine the effect of removing the standard on varying proportions of trials. If the standard is removed on all trials, we have the typical identification (absolute judgment) experiment. Comparison of performance in the typical delayed comparison and identification paradigms with the stimulus values constant would give us some information as to the role of the standard. Presentation of varying proportions of both types of trial will give more information, since it enables us to determine the basis of the differences between the two paradigms.

Some studies have compared auditory amplitude discrimination performance on delayed comparison and identification trials. Pollack (1954) examined amplitude discrimination thresholds under a variety of experimental conditions. He found that the probability of detecting a stimulus change was a function of both the availability of a reference standard and the stability of the standard signal; both variables improved performance. He failed to find any significant deterioration in performance with increases in the interstimulus interval. This does not, however, necessarily mean that the trace mode was not operating, since he used a coarse measure (DL) and short-duration standards $(50 \mathrm{msec})$. Other research by this author (Long, 1971) suggests that both acquisition and memory processes would be operating in silent intervals after such short tones, preventing a simple decay in performance.

Viemeister (1970) studied the effect of including a constant cue in what was described to the 0 as an identification task. He found that the presence of such a cue did not improve auditory intensity discrimination. This, unlike Pollack's work, would seem to support the claim that context coding is used in fixed-standard experiments. An analysis of intermediate conditions in which the standard is present on some but not all trials may help solve this apparent contradiction.

In another study, Pollack (1956) compared performance in delayed comparison and identification paradigms with stimulus values the same in both experiments. He found that performance in both tasks deteriorated as the range over which the stimuli could vary was increased. This could be taken as evidence that the context mode is used even in roving-standard discrimination experiments, an unlikely conclusion. He also noted, however, that the deterioration of performance with increase in the range of levels taken by 
the standard in the discrimination experiment was not entirely due to random factors. Response bias was directly related to the scale position of the standard stimulus. The $O$ had a tendency to judge the second tone as more intense than the first when it was low. Such effects increased with increases in range. This could account in part for the effect of range on the discrimination experiment. It is also possible that the acquisition process would demand more time when a roving standard is used. The stimuli used were only $100 \mathrm{msec}$ in duration. so that if acquisition was altered by the use of a roving standard. this. rather than greater memory loss, could account for the performance decrements.

This evidence. that response bias is linked to the level of the standard in roving-standard experiments. is consistent with predictions from adaptation level theory (Helson. 1964) that suggest that even when a standard is present it does not completely determine the basis for the sensory comparison but is merely the most heavily weighted stimulus in a weighted mean of all the stimuli presented. It also suggests that Es who average performance over all levels of a roving-standard experiment are confounding noise due to acquisition, criterion. memory, and sensitivity variance. This provides another reason for attempting to study the standard by removing it on varying proportions of the trials. If we have some understanding of the role of the standard in the fixed-standard paradigm. the introduction of the above problems by using the roving-standard paradigm. in an attempt to ensure that the $O$ is using the standard, may no longer be necessary.

\section{EXPERIMENT I}

This experiment is designed to examine the role of the "standard" stimulus when an O compares two successively presented auditory stimuli in a discrimination experiment. This was done by forcing $O$ to utilize an intertrial long.term memory for the standard on some trials by withholding the standard on varying proportions of the trials.

\section{Method}

\section{Apparatus and Procedure}

The stimuli were $1.000-\mathrm{Hz}$ pure tones generated by an oscillator (Hewlett-Packard 2048) and gated through electronic switches (Grason-Stadler 829) with rise and decay times of $10 \mathrm{msec}$. They were presented simultaneously through headphones (Permaflux PDR 8,600-ohn impedance) to three Os in separate acoustic testing chambers (IAC Model 102608). In terms of the RMS voltage from a 600-ohm impedance source. the first (standard) tone burst was a constant $120 \mathrm{mV}$ and the second (comparison) tone burst was either 120 or $107 \mathrm{mV}$. These amplitudes were arbitrarily chosen to be both comfortably audible (approximatel 51-52 dB SPL) and sufficiently difterent in anplitude (approsimately $\mathrm{I} \mathrm{dB}$ ) to produce about $80^{\circ}$; correct responding during 5 days of prelinunary testing.

Visual cues were presented to the $O$ on a CRT display
(Tektronix 609) located on a table in front of him. Responses were made through push buttons located next to the display. The electronic switihes and visual cues were under the control of a DEC PDP-12 computer, which also recorded responses.

Each complete trial cycle contained seven successive phases: a standard tone, an interstimulus interval, a comparison tone. a response period, a feedback period, and an intertrial interval. The intervals containing the standard and comparison tones were delineated for the $O$ by the illumination of specified points on the CRT display. Appropriate words were displayed for the warning period, the response period, and the feedback period. as timing was less critical during these phases.

The warning period lasted $500 \mathrm{msec}$ and was followed immediately by a 500-msec interval normally containing the standard tone. The 500-msec comparison tone followed after a silent interstimulus interval, which was also $500 \mathrm{msec}$. These durations were chosen because previous experimentation (Long, 1971) had indicated that stimulus acquisition is complete and memory decay moderate at these durations. The $O$ was given $2 \mathrm{sec}$ after offset of the second tone to indicate, by pressing an appropriate push button, one of two decisions: "Both tone bursts were of the same amplitude," or "The second tone burst "as softer than the first." The response was immediately visible on the CRT as a number, which remained on while the correct response was displayed during a subsequent $500-\mathrm{msec}$ feedback period. Each $O$ could see his response alone. Finally, there was an intertrial interval of $500 \mathrm{msec}$ before the start of the next trial.

The sequence of stimuli was determined in blocks of 100 trials, with the second stimulus having the same amplitude as the first on a randomly determined 50 out of these 100 trials and differing on the remaining 50. Each experimental session consisted of six 100-trial blocks separated by a 1 -min rest period. Two conditions were measured in each session. The first block in each condition (the first and fourth blocks in the session) served as unrecorded practice trials. There were six conditions defined by the proportion of trials within each 100-trial block containing the standard: $0, .2, .4, .6, .8$, or 1.0 . The trials on which the standard was absent were equally distributed over "same" and "sof ter" trials. Note that the two extreme conditions ( 0 and 1.0$)$ are the typical "identification" and "fixed-standard comparison" paradigms, respectively.

All Os were given five preliminary training session, in which the standard was always present. Each condition was then presented four times, twice in each half of the session. The order of presentation of the conditions was pseudorandomized across sessions and was unknown to the $O$. One session was conducted each day.

Os were three male students from Princeton High School. They were paid $\$ 2$ for each 55 -min session. Each O was fully informed of the physical nature and relative frequency of the stimuli and of the random method for generating the stimulus sequences.

The signal detection theory measure. d' (Green \& Swets. 1956), was used as the measure of performance. This measure. which is relatively independent of response bias. expresses performance as a $z$ score of the physical separation of the stimulus patterns expressed in terms of the assumed variances of the sensory representation of each stimulus pattern. If these variances are assumed to be equal, estimates of $d^{\prime}$ can be obtained by locating obtained estimates of the probabilities that the $\mathrm{O}$ correctly [hit rate, $\operatorname{Pr}(\mathrm{H})$ ] and incorrectly [false alarn rate. $\operatorname{Pr}(\mathrm{F} \cdot \mathrm{A})]$ identified as the stimuli as the "same" in tables given by Elliot (1965). Estimates of statistical significance were. howerer, based on chi-square analyses of the hit and false alarm rates wherever possible, because such an analysis makes use of more of the data.

\section{Results}

Estimates of $d^{\prime}$ for each $O$ under each condition are 
Table 1

Estimates of $d^{\prime}$ for Each $O$ and the Mean for All Os for All Conditions of Experiment I

\begin{tabular}{|c|c|c|c|c|c|c|c|c|}
\hline \multirow{2}{*}{$\begin{array}{c}\text { Probability } \\
\text { of } \\
\text { Standard }\end{array}$} & \multicolumn{2}{|c|}{ O A } & \multicolumn{2}{|c|}{ O B } & \multicolumn{2}{|c|}{ OC } & \multicolumn{2}{|c|}{ Mean } \\
\hline & $\begin{array}{c}\text { Standard } \\
\text { Present }\end{array}$ & $\begin{array}{c}\text { Standard } \\
\text { Absent }\end{array}$ & $\begin{array}{c}\text { Standard } \\
\text { Present }\end{array}$ & $\begin{array}{c}\text { Standard } \\
\text { Absent }\end{array}$ & $\begin{array}{c}\text { Standard } \\
\text { Present }\end{array}$ & $\begin{array}{c}\text { Standard } \\
\text { Absent }\end{array}$ & $\begin{array}{c}\text { Standard } \\
\text { Present }\end{array}$ & $\begin{array}{c}\text { Standard } \\
\text { Absent } \\
\end{array}$ \\
\hline $\begin{array}{r}0 \\
.2 \\
.4 \\
.6 \\
.8 \\
1.0\end{array}$ & $\begin{array}{l}2.33 \\
2.40 \\
2.63 \\
2.46 \\
2.83\end{array}$ & $\begin{array}{l}2.22 \\
1.87 \\
1.80 \\
1.97 \\
1.84\end{array}$ & $\begin{array}{l}1.36 \\
2.12 \\
2.01 \\
2.12 \\
2.12\end{array}$ & $\begin{array}{l}1.83 \\
1.32 \\
1.58 \\
1.48 \\
1.20\end{array}$ & $\begin{array}{r}.88 \\
1.10 \\
.87 \\
.92 \\
1.12\end{array}$ & $\begin{array}{l}.82 \\
.61 \\
.94 \\
.38 \\
.52\end{array}$ & $\begin{array}{l}1.52 \\
1.87 \\
1.84 \\
1.83 \\
2.02\end{array}$ & $\begin{array}{l}1.62 \\
1.27 \\
1.44 \\
1.28 \\
1.19\end{array}$ \\
\hline
\end{tabular}

presented in Table 1 and Fig. 1, along with the average across all Os. In general, the Os were more likely to be correct at any particular probability of presentation when a standard stimulus was present than when it was absent $(p<.01)$. Further, the probability of being correct on trials containing the "standard" was directly related to the proportion of such trials within a session $(p<.01)$; similarly, the probability of a correct response on "no-standard" trials was directly related to the proportion of that type of trial within each block $(p<.01)$. Of particular interest is the large decrement in accuracy produced when the standard is omitted during those sessions when it almost always occurs (e.g., the 6 and .8 conditions in Fig. 1).

\section{Discussion}

The importance of the standard was related to how confident the Os could be that it would occur. While they did better on trials containing a standard regardless of the proportion of such trials, their accuracy on both "standard-present" and "standard-absent" trials was positively related to the probability of that particular trial. This seems to suggest that the $O$, as predicted in the introduction, operates in one of two different memory comparison modes, depending on the stimulus pattern. The mode used when a standard is available ("trace" mode) is superior to that used when the standard is absent ("context" mode). Performance under either mode is optimal only when the $O$ is prepared to utilize that mode.

Viemeister (1970) found no difference between standard always present and standard always absent conditions. This apparent conflict with the above study may be due to differences in the instructions given to the Os. Viemeister's $O$ (N.V.) was instructed for an identification task and thus may not have perceived the optimal comparison strategy as relevant, even when the standard was present.

\section{EXPERIMENT II}

It was suggested in the above discussion that the O's expectation of, and preparation for, a specific type of trial determines how well he operates in the appropriate memory mode. This may be due to an inability to switch from one mode to another within a series of trials, or to his inability to predict which mode is appropriate for
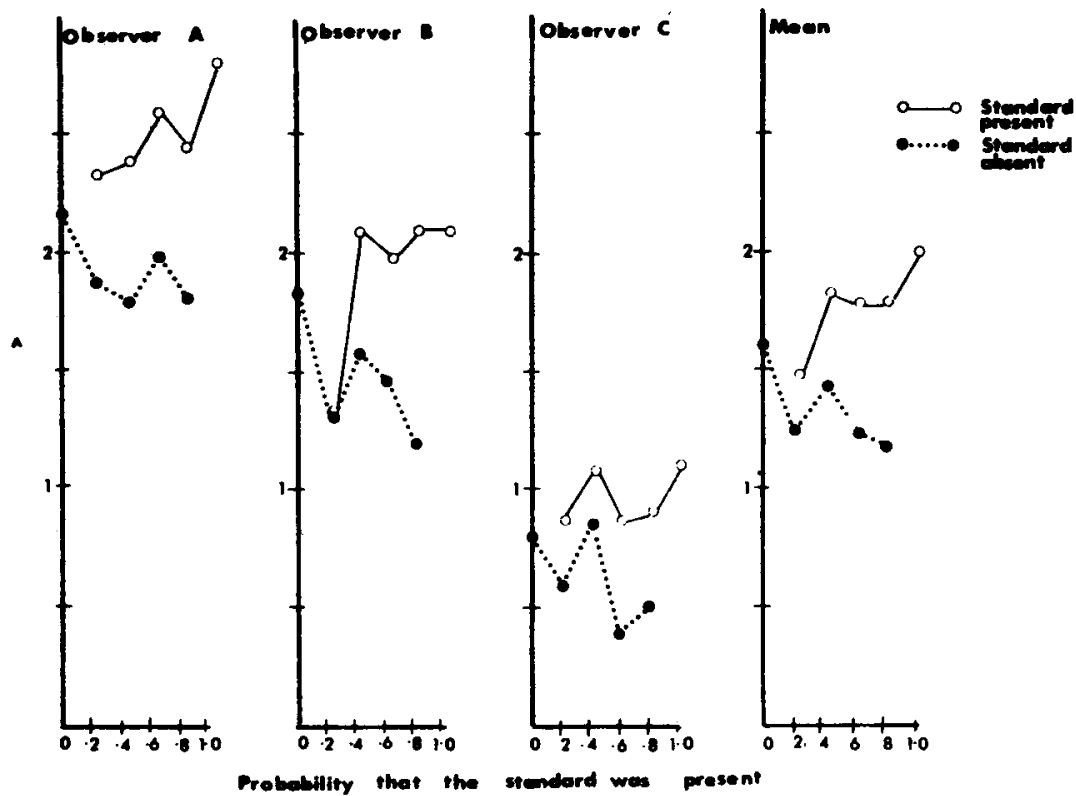

Fig. 1. Performance, $\hat{d}^{\prime}$, with the standard present and with it absent for all conditions in Experiment 1. 
Table 2

Estimates of d' for Os Trained on "Comparison" and "Identification" Trials in Experiment II

\begin{tabular}{|c|c|c|c|c|c|c|c|c|}
\hline \multirow{3}{*}{$\begin{array}{c}\text { Probability } \\
\text { of } \\
\text { Standard }\end{array}$} & \multicolumn{8}{|c|}{ Comparison Trained } \\
\hline & \multicolumn{2}{|c|}{$\mathrm{OCl}$} & \multicolumn{2}{|c|}{$\mathrm{OC2}$} & \multicolumn{2}{|c|}{$0 \mathrm{C} 3$} & \multicolumn{2}{|c|}{ Mean } \\
\hline & $\begin{array}{c}\text { Standard } \\
\text { Present }\end{array}$ & $\begin{array}{c}\text { Standard } \\
\text { Absent }\end{array}$ & $\begin{array}{c}\text { Standard } \\
\text { Present }\end{array}$ & $\begin{array}{c}\text { Standard } \\
\text { Absent }\end{array}$ & $\begin{array}{c}\text { Standard } \\
\text { Present }\end{array}$ & $\begin{array}{c}\text { Standard } \\
\text { Absent }\end{array}$ & $\begin{array}{c}\text { Standard } \\
\text { Present }\end{array}$ & $\begin{array}{c}\text { Standard } \\
\text { Absent }\end{array}$ \\
\hline 0 & & 1.48 & & 1.28 & & 1.13 & & 1.30 \\
\hline .2 & 1.76 & 1.21 & 1.45 & 1.18 & 1.46 & .66 & 1.56 & 1.02 \\
\hline .4 & 1.83 & 1.00 & 1.48 & .94 & 1.49 & .80 & 1.60 & .91 \\
\hline .6 & 1.57 & 1.16 & 1.62 & .91 & 1.44 & .72 & 1.54 & .93 \\
\hline .8 & 1.94 & 1.26 & 1.88 & 1.12 & 1.62 & .66 & 1.81 & 1.01 \\
\hline \multirow[t]{3}{*}{1.0} & 1.90 & & 2.20 & & 1.54 & & 1.88 & \\
\hline & \multicolumn{8}{|c|}{ Identification Trained } \\
\hline & \multicolumn{2}{|c|}{ O I1 } & \multicolumn{2}{|c|}{ O I2 } & & & \multicolumn{2}{|c|}{ Mean } \\
\hline 0 & & 1.50 & & 1.31 & & 2.52 & & 1.78 \\
\hline .2 & 2.16 & 1.46 & 1.28 & .94 & 2.68 & 2.35 & 2.04 & 1.58 \\
\hline .4 & 2.14 & 1.46 & 1.56 & 1.18 & 2.95 & 2.42 & 2.22 & 1.69 \\
\hline .6 & 2.31 & 1.20 & 1.58 & 1.14 & 3.22 & 2.32 & 2.37 & 1.55 \\
\hline .8 & 2.03 & 1.02 & 1.45 & .98 & 3.04 & 2.33 & 2.17 & 1.44 \\
\hline 1.0 & 2.27 & & 1.44 & & 3.19 & & 2.30 & \\
\hline
\end{tabular}

each trial. If the latter interpretation is true, it should be possible to eliminate the effect by giving the Os information at the start of each trial which enables them to predict whether the standard will be present or absent.

It is also of interest to determine whether the superior performance on the comparison trials in Experiment 1 was due to a real difference in the trials or simply to the Os' pretraining with that type of trial.

These two variables were examined by: repeating Experiment I with two groups of Os (one group given initial training experience with "comparison" trials and the other with single-stimulus "identification" trials), and enabling the Os to predict the nature of the trials on half the sessions.

\begin{abstract}
Method
Procedure

The experimental method was essentially the same as in Experiment I, with the following exceptions. The interval between the trials was $1 \mathrm{sec}$ longer, so only four blocks of 100 trials of the same experimental condition were presented each session; the first 100 were unrecorded practice trials. Two sessions, separated by $1 / 2 \mathrm{~h}$, were given each day. In half of the sessions, the extra second between trials was used to cue the $O$ as to whether the standard would be present or absent on that trial by displaying either "present" or "absent" on the CRT display.

The Os were six nonstudent females (ages 20-30 years) from the Princeton area. Three were given six sessions of preliminary training with the standard present, and three were given six sessions with the standard absent. The three Os who were trained without the standard present were asked to respond "louder" and "softer" instead of "same" and "softer."
\end{abstract}

Fig. 2. Performance, $\dot{d}^{\prime}$, with the standard present and standard absent for "comparison" (standard present) trained Os in Experiment II.
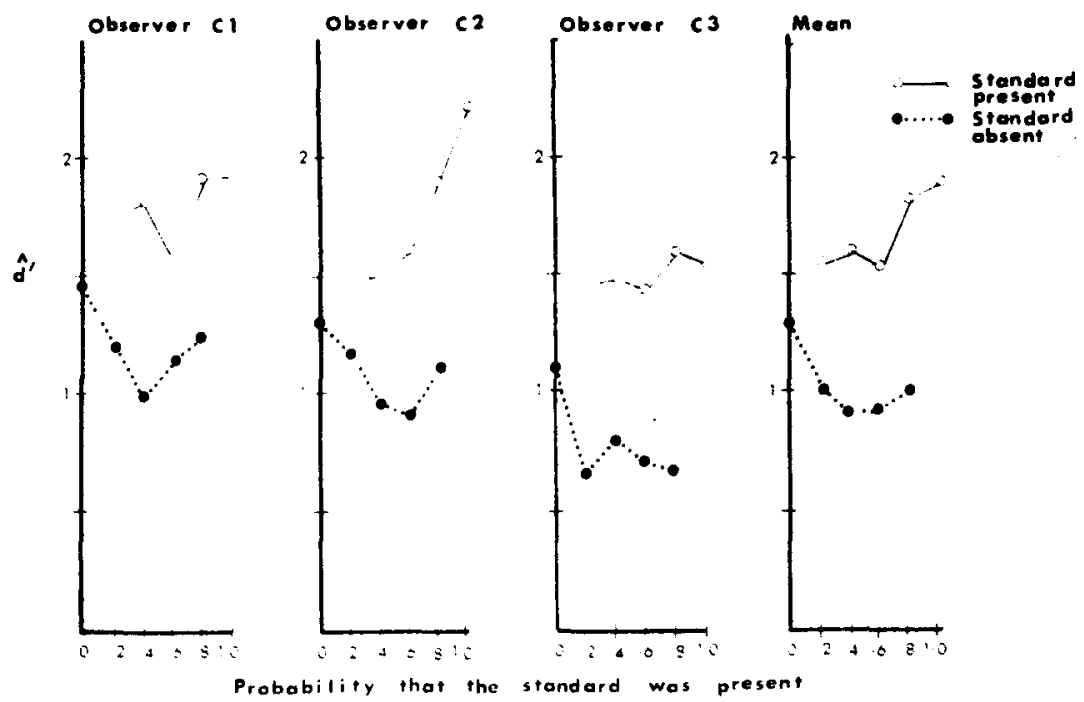


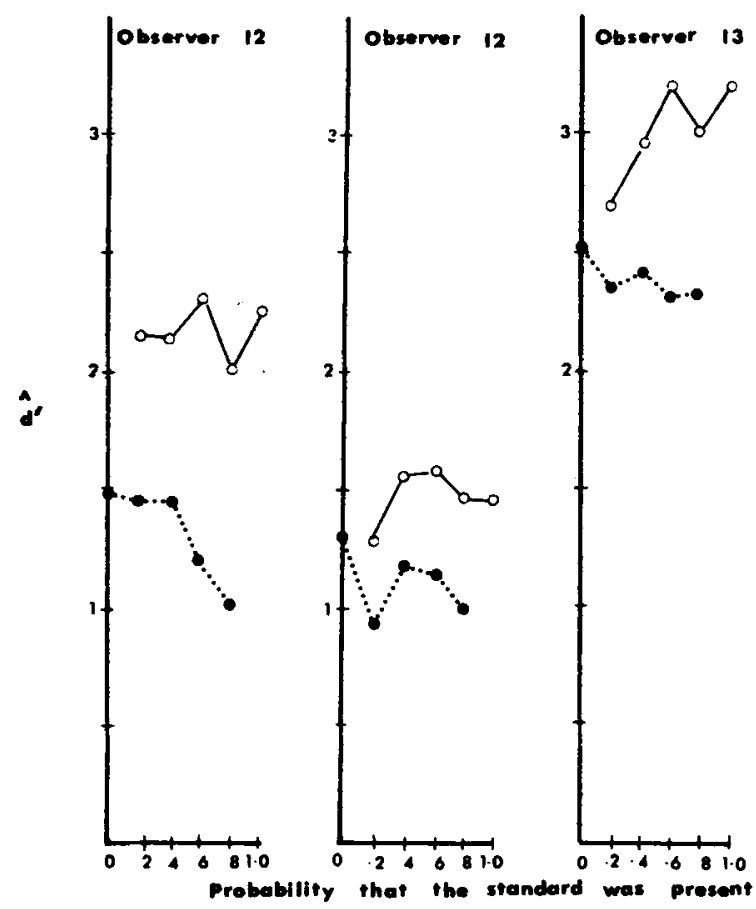

\section{Results}

A chi-square analysis of the hit and false alarm rates for each $\mathbf{O}$ demonstrated that enabling the $O$ to predict the nature of each trial did not have a statistically significant effect $(p>.05)$. Since the null hypothesis could not be rejected, the data from the two information (cue) conditions were combined. Table 2 presents estimates of $d^{\prime}$ for all conditions for each of the Os in the two pretraining groups, along with the means for each group. The data is presented graphically in Figs. 2 and 3.

All Os show the same general pattern of performance as those in Experiment $I$, i.e., performance is always optimal when the standard is present and performance on either type of trial tends to deteriorate as the probability of that type of trial decreases. The nature of the initial training had no consistent effect on performance. As differences in the overall level of performance are probably due to individual differences, the amount of change in performance when the standard is withheld provides a better estimate of any difference between the groups. A Mann-Whitney $U$ test of the mean change for each $O$ in both training groups failed to show any significant effect of training $(p>.05)$.

\section{Discussion}

Neither enabling the Os to predict the nature of each trial nor providing pretraining with one particular type of trial altered the Os' performance. The Os still performed better when a standard was present than when it was absent, and performance on either type of

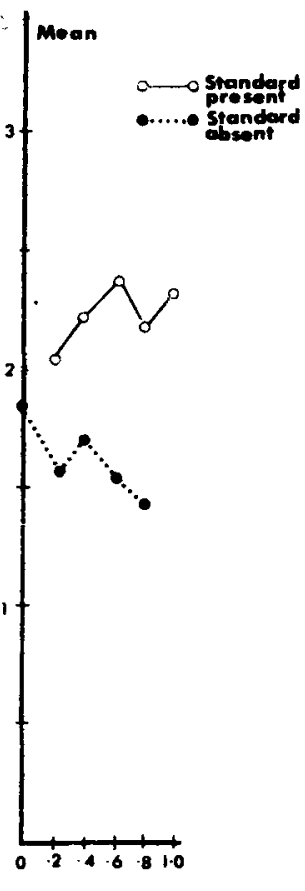

Fig. 3. Performance, $\hat{d}^{\prime}$, with the standard present and standard absent for "identification" (standard absent) trained Os in Experiment II. trial still improved as that type of trial became more probable. This suggests that the Os were, in fact, using a different, and somewhat superior, comparison mode when the standard was present than when it was absent and that they found it difficult to switch modes within a session even when they knew what type of trial was going to be presented.

Durlach and Braida (1969) claim that the context mode will be used in any fixed-standard experiment in which the range is small. This claim is not consistent with the findings of Experiments $I$ and II, that performance on trials when the standard was present was superior to performance on trials when it was absent. One way to reconcile such a difference with Durlach and Braida's claim would be to say that the standard merely provided additional information about the total stimulus context and was not used as a basis for comparison. Another stems from adaptation level theory, which suggests that the more frequently the standard is presented, the greater the difference between the adaptation level (a weighted mean of all stimuli) and the comparison stimulus, and so the easier the discrimination. The deterioration in performance on standard-absent trials during those sessions with a high probability of standard-present trials counters both such explanations, as any such additional information, or increase in adaptation level, should aid performance on both types of trial unless different modes are being used (separate AL may be developed for each mode). This evidence, thus, suggests that even in fixed-standard experiments, the trace mode is used when the standard is present.

The lack of any differences between identification- 
Table 3

Estimates of Response Bias (Probability of Saying "Same") as a Function of the Stability and Level of the Standard Os

\begin{tabular}{lllllll}
$\mathrm{C} 1$ & $\mathrm{C} 2$ & $\mathrm{C} 3$ & $\mathrm{I}$ & 12 & 13 & $\overline{\mathrm{X}}$ \\
\hline
\end{tabular}

Experiment II

Fixed Standard

$\begin{array}{llllllll}\text { (Low) } & .50 & .50 & .63 & .53 & .54 & .50 & .53\end{array}$

Experiment II

Random Standard

(Low)

$\begin{array}{lllllll}.43 & .47 & .52 & .47 & .40 & .49 & .46\end{array}$

Random Standard (High)

$\begin{array}{lllllll}.62 & .63 & .84 & .65 & .67 & .55 & .66\end{array}$

and comparison-trained $O s$ in this experiment invalidates the previous suggestion for the difference between the author's results and those of Viemeister. The Os in the studies reported in this paper experienced conditions in which the standard was present on some of the trials. Such exposure may have meant that even the identification-trained Os were exposed to the advantages of the comparison or trace mode and hence were more prepared to use it. Viemeister's one $\mathrm{O}$ (N.V.) had no experience with such intermediate conditions and thus may have stayed with the identification strategy as instructed. In a task like this, where more than one strategy is possible, it would not be surprising to find one $O$ who sticks with one strategy even when it is not the optimal one.

Most signal detection theorists have claimed that attitudinal and instructional variables influence response bias ("criterion") rather than sensitivity. The deterioration in performance on either type of trial with a decrease in the probability of that type of trial was,
Fig. 4. Response bias (probability of saying "same") as a function of the stability and level of the standard.
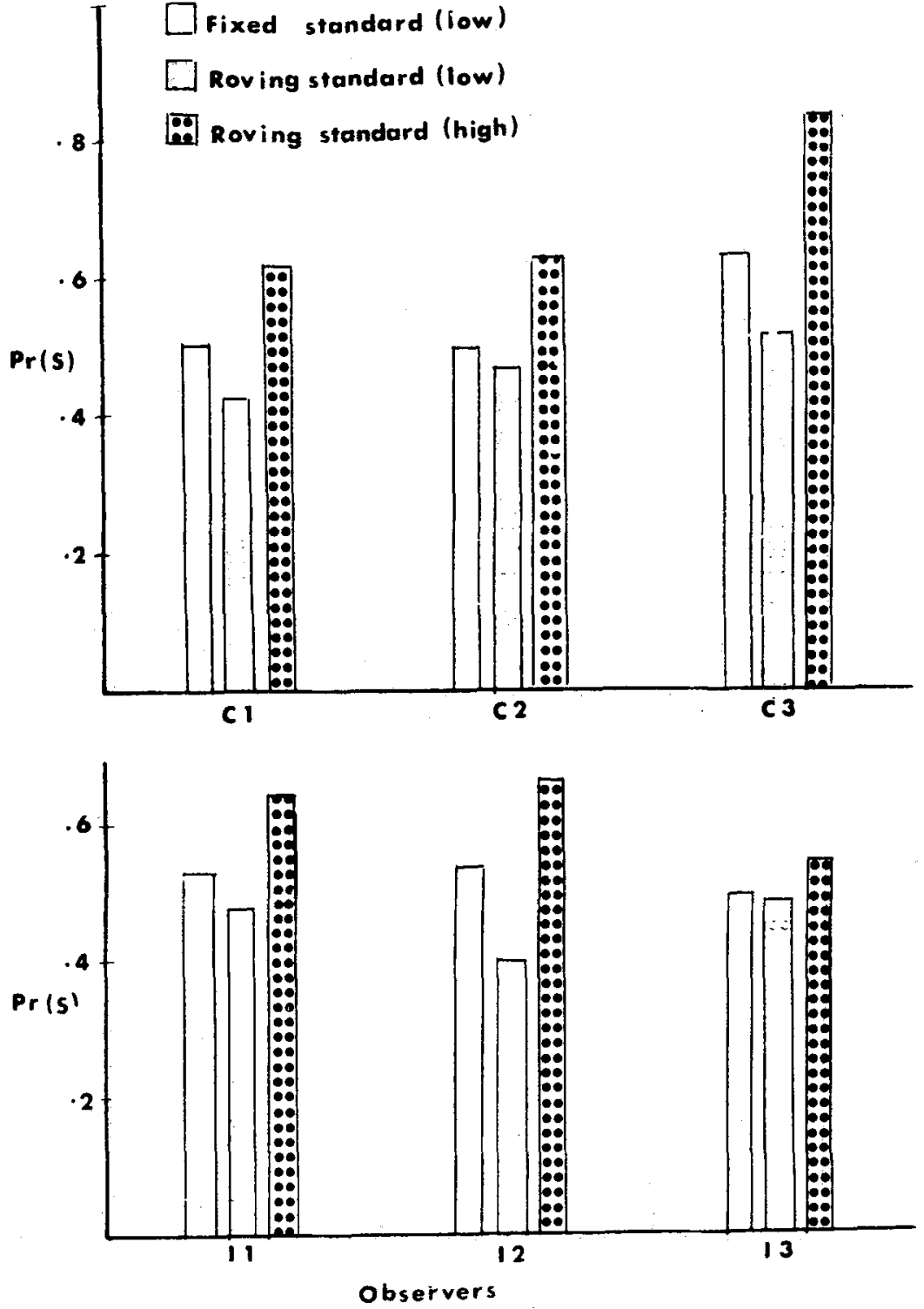
Table 4

Performance, d', as a Function of the Stability and Level of the Standard

\begin{tabular}{lllllllll}
\hline $\mathrm{C} 1$ & $\mathrm{C} 2$ & $\mathrm{C} 3$ & $\mathrm{I1}$ & $\mathrm{I} 2$ & 13 & $\overline{\mathrm{X}}$ \\
\hline
\end{tabular}

Experiment I

Fixed Standard

(Low)

$\begin{array}{lllllll}1.51 & 1.36 & 1.17 & 1.71 & 1.27 & 2.75 & 1.63\end{array}$

Experiment II

Randon Standard

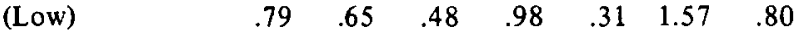

Random Standard

(High)

$\begin{array}{lllllll}.85 & 1.23 & .76 & 1.07 & .57 & 1.86 & 1.06\end{array}$

however, a drop in sensitivity. This suggests that the actual processing of stimuli can be effected by these cognitive variables. Sperling (1967) has shown this to be true for complicated visual displays; instructions given after stimulus offset but before stimulus processing was complete determined what type of information was processed. This experiment suggests that processing can be independent of the information provided on each trial but dependent on the Os' overall expectation or mode of processing.
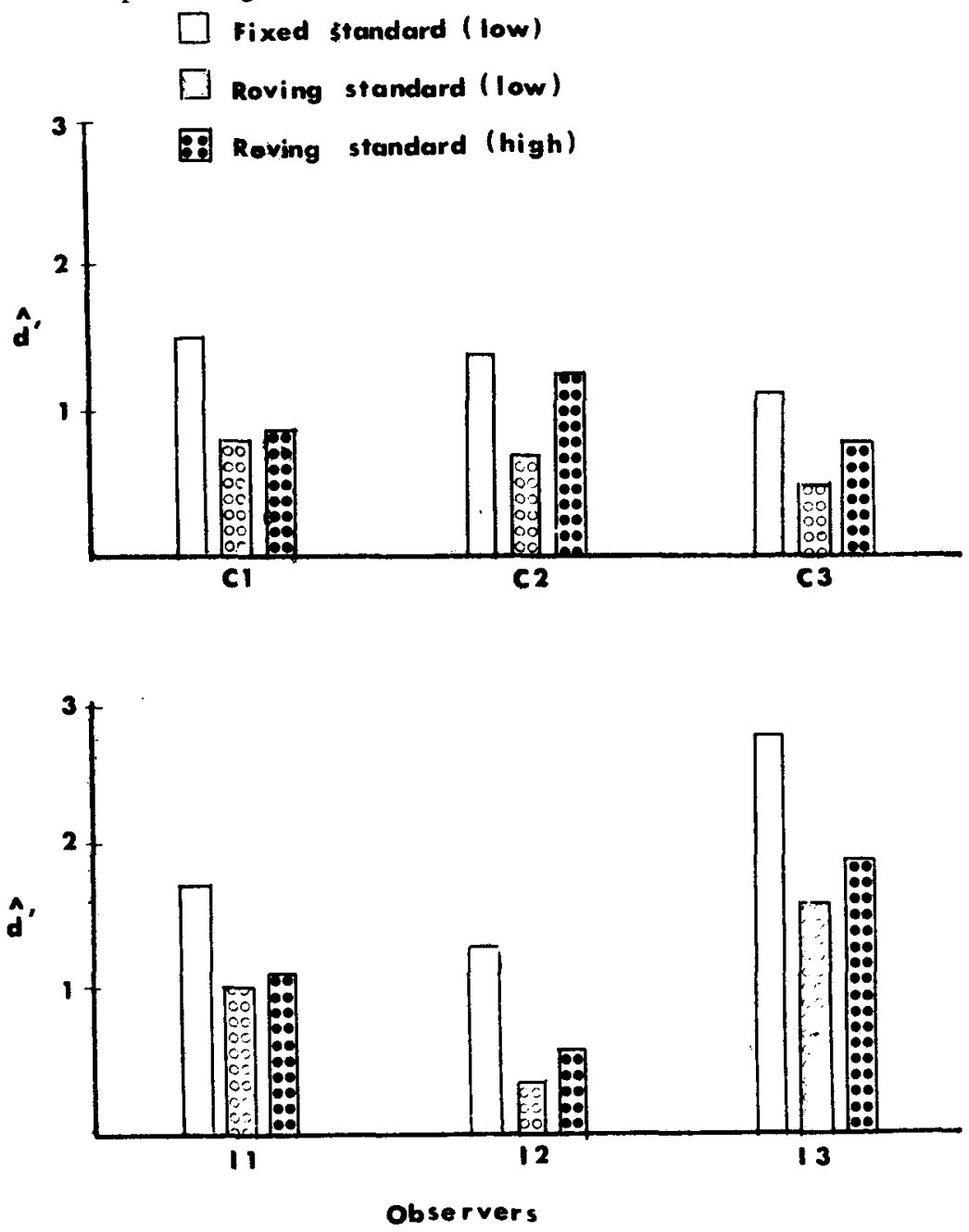

\section{EXPERIMENT III}

It has been claimed that randomly varying the overall level of the pair of tones constituting a single trial (roving-standard paradigm) prevents the Os from obtaining a long-term memory of the standard and thus ensures that they make their comparisons with the short-term memory of the standard presented on that particular trial. This experiment examines a minimal form of this paradigm in which the tone pair varies randomly between two different levels. Experiment Il was repeated with the tone pair able to take a second (louder) level on half the trials within each block.

\section{Method}

\section{Procedure}

The procedure was the same as in Experiment II. except that on a randomly chosen half of the trials within each block, the amplitude of the pair of tones was inereased. In terms of the RMS voltage from the $600-0 h m$ source the standard was $300 \mathrm{mV}$. The variable was about $270 \mathrm{mV}$. This stimulus difference was obtained by putting the $300-\mathrm{mV}$ pulse through the same attenuator as the $120-\mathrm{mV}$ pulse. This meant that there
Fig. 5. Performance, $\hat{d}^{\prime}$, as a function of the stability and level of the standard. 
Fig. 6. Averaged performance, $\hat{d}^{\prime}$, for all os for fixed-and roving-standard experiments.
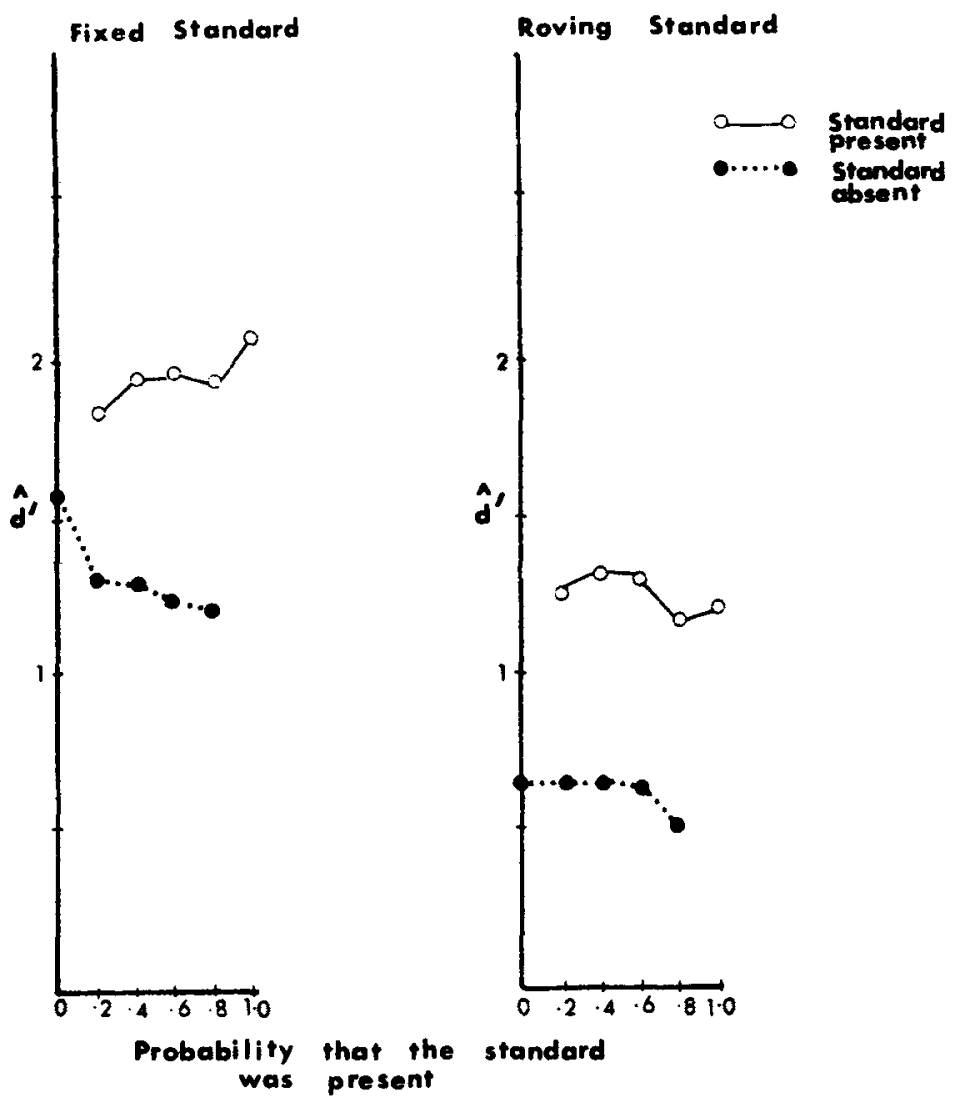

was approximately an 8- $\mathrm{dB}$ difference between standards and a 1-dB increment.

The six Os from Experiment II were aiso used in this experiment. The two experiments were run concurrently, i.e., the two experiments were run on randomly assigned day's over a 5-week period. This was done to increase the comparability of the results.

\section{Results}

Estimates of response bias [the probability that the 0 said "same," $\operatorname{Pr}(S)]$ were obtained for each $O$ in the fixed-standard experiment (Experiment II) and for both levels of the standard in the roving-standard experiment (Experiment III). These estimates are presented in Table 3 and Fig. 4. When the standard was constant, little response bias was exhibited: most Os responded "same" about $50 \%$ of the time. The introduction of a higher level tone pair led to a decrease $(p<.001)$ in the probability that the $O$ would respond "same" when the lower level tones (the same tones as in the fixed-standard experiment) were presented and to an increase $(p<.001)$ in the same probability when the higher tones were presented. Because of the large difference in response bias (the Os said "same" an average of 66\% of the time for the high-amplitude tones in contrast with $46 \%$ of the time for the low-amplitude tones. a 20\% difference). estimates of ' $d$ ' were obtained separately for the high and low tones. Any further analysis was done by combining these separate estimates.
The effect on sensitivity of introducing a second level of tones can be seen in Table 4 and in Fig. 5, which present estimates of the Os' sensitivity in the fixed-standard experiment and for both levels of the roving-standard experiment. These estimates were obtained by averaging the estimates of $\mathrm{d}^{\prime}$ obtained in all other conditions. Sensitivity was always best in the fixed-standard experiment and worst for the same stimuli in the roving-standard experiment; the average d' from the latter condition was half that from the former. Performance on the higher amplitude tone pair was better than on the lower tone pair in the roving-standard experiment but lower than that in the fixed-standard experiment. A Friedman two-way analysis of variance of ranks shows these trends to be significant $(p<.001)$.

Figure 6 shows estimates of sensitivity with the standard present and with the standard absent for the fixed-standard and roving-standard experiments. All six Os showed similar patterns of results, so estimates of d' were obtained by averaging across Os and conditions. The same data are presented numerically in Table 5. Even though the overall superiority of comparison trials remains. the decrease in performance on either type of trial with the decrease in its probability of occurrence is no longer evident.

\section{Discussion}

The introduction of a higher amplitude tone pair. 
Table 5

Performance, d', in Fixed and Roving Standard Experiments as a Function of the Probability that the Standard was Presented

\begin{tabular}{|c|c|c|c|c|}
\hline \multirow{2}{*}{$\begin{array}{c}\begin{array}{c}\text { Probability } \\
\text { of } \\
\text { Standard }\end{array} \\
\end{array}$} & \multicolumn{2}{|c|}{ Fixed Standard } & \multicolumn{2}{|c|}{ Roving Standard } \\
\hline & $\begin{array}{c}\text { Standard } \\
\text { Present }\end{array}$ & $\begin{array}{c}\text { Standard } \\
\text { Absent }\end{array}$ & $\begin{array}{c}\text { Standard } \\
\text { Present }\end{array}$ & $\begin{array}{c}\text { Standard } \\
\text { Absent }\end{array}$ \\
\hline 0 & & 1.57 & & .64 \\
\hline .2 & 1.83 & 1.31 & 1.26 & .64 \\
\hline .4 & 1.94 & 1.31 & 1.31 & .64 \\
\hline .6 & 1.96 & 1.24 & 1.28 & .63 \\
\hline .8 & 1.94 & 1.21 & 1.16 & .49 \\
\hline 1.0 & 2.09 & & 1.19 & \\
\hline
\end{tabular}

changing the experiment from a fixed-standard paradigm to a roving-standard paradigm, led to response bias tied to the level of the standard and to an overall deterioration in performance. It did not change the relative superiority of performance on trials with a standard present over trials with it absent, but did eliminate the improvement in performance on either type of trial as the probability of that type of trial increased.

The finding that response bias in the roving-standard experiment was dependent on the level of the standard is consistent with Pollack's (1956) results, and the predictions of adaptation level theory. Most studies using the roving-standard paradigm combine the data from the different levels of the standard before obtaining estimates of performance. Such a procedure introduces an extra source of variance and may confound response bias with the other variables of interest.

The data from this experiment run counter to Durlach and Braida's assumptions. They assume that the introduction of a roving standard, i.e., an increase in stimulus range, should change performance only when the $\mathrm{O}$ is operating in the context mode. If this were true, and if, as demonstrated in the above studies, the $O$ is operating in the trace mode when a standard is presented, the drop in overall performance in Experiment III should have been limited to the standard-absent trials. This was not found; there seemed to be an equivalent drop in performance on both types of trial. It thus appears that the stimulus range interferes with performance under the trace mode as well as under the context mode. It could be hypothesized that this deterioration in performance with the increase in stimulus range might be independent of the memory process and be due to a deterioration in the acquisition process by which the $\mathrm{O}$ acquires his initial information about the stimulus. In a fixed-standard experiment, the $O$ expects the stimuli to be within a certain range, i.e., he has a long-term memory of the general amplitude of the tone. This may mean that he is able to obtain more information from the stimulus when it is presented to him. Whatever factor is causing the deterioration in performance in the roving-standard experiment, it appears to have interfered with the $O$ 's ability to perform maximally during sessions in which only one type of trial occurs, i.e., it seems to prevent him from making optimal use of either strategy. This suggests another hypothesis based on adaptation level theory; the greater range of stimuli presented in the roving-standard experiment means that the adaptation level is less fully determined by the standard and is, thus, more variable. This greater variability masks the lesser variability stemming from the probability of a particular type of trial and leads to an overall deterioration in performance.

The difference in performance with the high and low tone pairs in the roving-standard experiment probably stems from the experimental method. There was no attempt to ensure that the stimulus differences were equally discriminable for the two levels. The two tones were simply channeled through the same attenuator. This was done because it was convenient and because the change in performance on the lower tones, which were the same in both experiments, was of more interest. Limiting the number of levels the tone pair could take to two may limit the generality of the results, as may the arbitrary size of the stimulus increment. These variables need to be tested, but it seems unlikely that there will be any difference to the general form of the results.

As all the experiments reported in this paper used amplitude discrimination, no definite statement can yet be made about the role of the standard in pitch discrimination experiments. It is, however, hypothesized that the findings of these amplitude discrimination experiments will apply to pitch discrimination tasks because research into memory and acquisition processes in pitch and amplitude discrimination experiments (Kinchla \& Smyzer, 1967; Wickelgren, 1966, 1969; Massaro, 1970a, b; Long, 1971) suggest that similar processes are involved in both dimensions. This would mean that, contrary to the claims of Harris and Wickelgren, the comparison mode used will be a function of the availability of the standard as well as the number of levels it can take. This needs to be tested.

As the duration between the standard and the comparison stimulus increases, it is expected that performance with the standard present would deteriorate over time as the memory of the standard deteriorates. This would mean that the difference in performance between trials with the standard present and those with the standard absent should decrease until at some point there is no longer any difference. At this point, the $\mathrm{O}$ will assumably operate in the context mode for both conditions. Experiments need to be conducted which test this hypothesis and estimate the point at which the change occurs.

\section{SUMMARY AND CONCLUSIONS}

These studies support the hypothesis that the $O$ can 
operate in one of two modes when making an amplitude judgment about a tone. He can compare it with some trace of a recently presented standard tone or he can compare it with the overall context of amplitudes presented in the experiment. The mode used depends on the availability of a standard tone. Whenever such a tone is presented, the $O$ makes use of it, i.e., he operates in the trace mode. He can do this more easily when the standard is present on all trials, and he does not need to switch modes within a series of trials. Similarly, he can perform best in the context mode when the standard is never present. These results were not affected by the type of experience available to the $\mathrm{O}$ or by enabling him to predict the nature of each trial within a series.

The introduction of a second pair of tones of higher amplitude transforming a fixed-standard experiment into a roving-standard experiment introduced considerable response bias which was tied to the stimulus level. The use of a measure of performance which is relatively independent of response bias showed that there was also an overall deterioration in performance. This suggests that the roving-standard paradigm does not, as hypothesized by Harris, by Wickelgren, and by Durlach and Braida, favor either mode of comparison, but interferes with the 0 's capacity to obtain a clear representation of the stimuli.

The main implications of this study are that there is no need to use a roving-standard paradigm to insure that the $O$ is using the standard as the basis for his comparison and that use of such a paradigm may introduce variance due to response bias. impair overall performance, and prevent the O's making optimal use of either comparison mode.

\section{REFERENCES}

Durlach, N. L., \& Braida, L. D. Intensity perception: I. Preliminary theory of intensity resolution. Journal of the Acoustical Society of America, 1969, 46, 372-383.

Elliot, P. B. Tables of d'. In J. A. Swets (Ed.). Signal detection and recognition by human obsenvers. New York: Wiley, 1964.

Green, D. M., \& Swets, J. A. Signal detection theory and psychophysics. New York: Wiley, 1966.

Harris, J. D. The decline of pitch discrimination with time. Journal of Experimental Psychology, 1952, 43, 69-99.

Helson, H. H. Adaptation level theory. New York: Harper \& Row, 1964.

Kinchla, R, A., \& Smyzer, F. A. A diffusion model of perceptual memory. Perception \& Psychophysics. 1967. 2. 219-229.

Long, G. R. Successive comparisons of auditory amplitudes. PhD thesis, Princeton L'niversity, 1971.

Massaro, D. W. Consolidation and interference in the perceptual memory system. Perception \& Psychophysics, 1970a. 7 , 153-156.

Massaro, D. W. Perceptual processes and forgetting in memory tasks. Psychological Review. 1970b. 77, 557-567.

Pollack, I. Intensity discrimination thresholds under several psychophysical procedures. Journal of the A coustical Society of America, 1954, 26, 1056-1057.

Pollack, I. Identification and discrimination components of elementary auditory displays. Journal of the Acanstical Society of America, 1956, 28, 906-909.

Sperling, G. Successive approximations to a model for short term memory. Acta Psychologica, 1967, 27. 285-292.

Viemeister, N. F. Intensity discrimination: Performance in three paradignis. Perception \& Psychophysics, 1970, 8. 417-419.

Wickelgren, $\mathrm{W}$. A. Consolidation and retroactive interference in short term recognition memory for pitch. Journal of Experimental Psychology, 1966, 72, 250-295.

Wickelgren, W. A. Associative strength theory of recognition memory for pitch. Journal of Mathematical Psychology, 1969. 6. 13-61.

(Received for publication January 7, 1972; revision received May 20, 1972.) 